Accepted on 12 January 2011 for publication in the

Journal of Wind Engineering and Industrial Aerodynamics

\title{
CFD modeling of pollution dispersion in a street canyon: Comparison between LES and RANS
}

\author{
Yoshihide Tominaga $^{\mathrm{a},{ }^{*}}$ and Ted Stathopoulos ${ }^{\mathrm{b}}$ \\ ${ }^{a}$ Department of Architecture and Building Engineering, Niigata Institute of Technology, 1719 \\ Fujihashi, Kashiwazaki, Niigata, 945-1195, Japan \\ ${ }^{b}$ Centre for Building Studies, Department of Building, Civil and Envir. Engineering, Concordia University, \\ 1455 de Maisonneuve Blvd West, H3G 1M8, Montreal, Canada \\ * Corresponding author:Tel\&Fax:+81-257-22-8176, E-mail address: tominaga@abe.niit.ac.jp (Y.Tom- \\ inaga).
}

\begin{abstract}
CFD modeling using RANS and LES of pollutant dispersion in a three-dimensional street canyon is investigated by comparison with measurements. The purpose of this study is to confirm the accuracy of LES in modeling plume dispersion in a simple street canyon model and to clarify the mechanism of the discrepancy in relation to RANS computation. Simple LES modeling is shown by comparison with wind tunnel experiments to give better results than conventional RANS computation (RNG) modeling of the distribution of mean concentration. The horizontal diffusion of concentration is well reproduced by LES, due mainly to the reproduction of unsteady concentration fluctuations in the street canyon.
\end{abstract}

\section{Keywords}

CFD, Pollutant dispersion, Street Canyon, LES, RANS

\section{$1 \quad$ Introduction}

Microclimate and air quality in urban areas has gained increasing attention in recent years. Street canyons are typical architectural structures in urban environments and they represent highly polluted zones around buildings. In order to minimize the effect of these pollutants in built-up environments, it is necessary to model and accurately predict contaminant dispersion properties in street canyons. Many fundamental studies have been conducted over the years by experimental modeling of flow and pollutant dispersion in street canyons using wind tunnels. Experimental techniques for modeling pollutant diffusion in street canyons were reviewed in detail by Vardoulakis et al. (2003). On the other hand, the rapid development of computer hardware and numerical algorithms has led to wide utilization of Computational Fluid Dynamics (CFD) in the study of wind fields and pollutant transport in urban street canyons see Li et al. (2006).

Reynolds-Averaged Navier-Stokes equation (RANS) models, in particular with the standard k- $\varepsilon$ model (hereafter SKE; Launder and Spalding, 1972), have been commonly adopted as turbulence models in calculating flow and dispersion in street canyons. Previously published papers have compared turbulence models for this type of flow. As target experimental data for validating CFD results, wind tunnel experiments on a twodimensional street canyon with $\mathrm{H} / \mathrm{W}=1.0$ (H: building height, $\mathrm{W}$; distance between buildings) obtained by Pavageau and Schatzmann (1999) have been used in several studies (Meroney et al., 1996). Chang and Meroney (2001) indicated that the concentrations obtained by SKE and the RNG k- $\varepsilon$ model (hereafter RNG; Yakhot et al., 1992) were similar, although both were overestimated in comparison with the experimental results. Furthermore, Chan et al. (2002) reported that the RNG model yields better agreement than other turbu- 
lence models, i.e. SKE and the Realizable model (Shih et al., 1995), in which concentration distributions at wall surfaces were evaluated higher than those of the experiment. This difference was attributed to the smaller eddy viscosity estimated by the RNG model compared with the other models. Nazridoust and Ahmadi (2006) analyzed the same configuration using the Reynolds stress model (hereafter RSM) and other turbulence models and reported that the RSM over-predicts the concentration at the leeward wall surface in high wind velocity cases, although it generally provides better agreement with the experiment on concentration distribution near the surface wall than the other models.

As a target for validating CFD applied to pollutant diffusion in continuous street canyons, MUST (Mock Urban Setting Test) observation data have often been used (Yee and Biltoit, 2004). A $12 \times 10$ aligned array of shipping containers was used to simulate the urban environment. Each container was $12.2 \mathrm{~m}$ long, $2.42 \mathrm{~m}$ wide and $2.54 \mathrm{~m}$ height. A detailed wind tunnel experiment was conducted for this configuration (Harms et al., 2005 ) and the model evaluation guidance and protocol document is available from the European COST action 732 (Britter and Schatzmann, 2007). Hanna et al. (2004) reported that the prediction results obtained by SKE were smaller than the observation data with an average concentration $36 \%$ lower and a maximum concentration 2 times lower. Milliez and Carissimo (2007) also conducted an analysis using SKE and compared these results with observations. They reported that there is a tendency to overestimate the turbulent energy in the case with low inflow velocity, leading to underestimation of concentration in the CFD results.

RANS models have been widely used for investigations of flow and dispersion in street canyons, although, as already discussed, several previous studies have pointed out some inherent limitations in the models. The subjects of these investigations include effects of canyon shape (Sini et al. (1996); Chan et al. (2001)), effects of turbulence intensity of approach flow (Kim and Baik (2003)), thermal effects due to sunshine (Sini et al. (1996), Xie et al. (2005)), and so on. Therefore, the relative performance of simulation methods when applied to the dispersion problem in street canyons should be studied in order to assess the reliability of these investigations.

Several studies have argued that LES results show good agreement with experimental results in terms of the distributions of mean velocity and turbulence energy around a simple building, even when the simple subgrid scale model is used (Murakami, 1993; Rodi, 1997; Tominaga et al., 2008a). This is because the momentum diffusion due to vortex shedding around the building, which is not reproduced in steady-state RANS computation, is closely related to the formation of the mean flowfield. The authors have confirmed the accuracy of LES in modeling dispersion near and around a simple building model and clarified the mechanism of the discrepancy in relation to the RANS computation in a previous study (Tominaga and Stathopoulos, 2010). In that study, it was reported that the RANS model underestimates the turbulence diffusion near a building in comparison to LES and the modeling accuracy of turbulent diffusion is very important for predicting the concentration distribution. Blocken et al. (2008) also pointed out that transient simulations might be required to achieve more accurate results through steady-state RANS computations of pollutant dispersion in the neutrally stable atmospheric boundary layer for several case studies. On dispersion in a street canyon, Walton et al. (2002a, $2002 \mathrm{~b}$ ) compared results obtained by LES and SKE with results of experiments conducted in a courtyard space surrounded by buildings, similar to a street canyon. Recently, Li et al. (2008) and Hu et al. (2009) applied LES to dispersion modeling in street canyons. According to their findings, LES is in close agreement with the experimental results, and they concluded that this is because LES predicts turbulence intensity more accurately. Santiago et al. (2010) also reported that for the MUST case, LES results are closer to the experimental values for the local vertical mean velocity than SKE results. However, very few studies have evaluated the basic performance of LES in modeling the dispersion field in a street canyon by comparison with the RANS model.

This study extends the investigation of flow and dispersion around a cubic building in a previous study (Tominaga and Stathopoulos, 2010) to flow and dispersion in a simple street canyon model. Its purpose is to confirm the accuracy of LES in modeling dispersion in a street canyon and to clarify the mechanism of the discrepancy in relation to the RANS computation.

\section{Computational details}

\subsection{Experimental setup}

Fig. 1 shows the model configuration. In the street canyon model adopted here, the building length is finite and the ambient wind blows perpendicular to the street canyon. Two parameters of canyon shape, $\mathrm{H} / \mathrm{W}$ and $\mathrm{H} / \mathrm{L}$, are defined as 1.0 and 0.5 , respectively ( $\mathrm{H}$ : building height, $\mathrm{W}$ : distance between buildings, and L: build- 
ing length). This geometry was expected to produce a skimming flow regime in the canyon (Hunter et al., 1992 ; Baik et al., 2003). Although this is a simple canyon configuration for investigating the basic structure of flow and dispersion in a street canyon, a parametric study with different aspect ratios would be of great interest in further study.

Experiments were carried out in the wind tunnel at Niigata Institute of Technology. The test section of the boundary layer wind tunnel is $13 \mathrm{~m}$ long, $1.8 \mathrm{~m}$ high and $1.8 \mathrm{~m}$ wide. A combination of spires and surface roughness is used in order to simulate an approach wind profile. The quantities at the inflow boundary are shown in Fig. 2. The vertical profile of mean velocity in the approaching flow approximately obeys a power law with an exponent 0.21 . The streamwise turbulent intensity at building height $\mathrm{H}$ is $20 \%$. Passive pollutant was released at a point source at the center of the street bottom. Ethylene $(\mathrm{C} 2 \mathrm{H} 4)$ was used as a tracer gas. The concentration of emission gas was $1000 \mathrm{ppm}$. The ratio of exit velocity, $\mathrm{W}_{\mathrm{S}}$ to $<\mathrm{u}_{\mathrm{b}}>$ was 0.12 , where $<\mathrm{u}_{\mathrm{b}}>=3.8$ $\mathrm{m} / \mathrm{s}$ is the upwind mean velocity at building height $\mathrm{H}$. Concentration measurements were performed using a high-speed total Hydrocarbon analyzer (Technica; H-THCA-01). Wind velocity was measured by a split fibre probe (Dantec Dynamics; 55R55) and a CTA module, which can discern three-dimensional components of velocity vector. The time averaging was conducted for a period of $30 \mathrm{sec}$ to obtain statistical values.

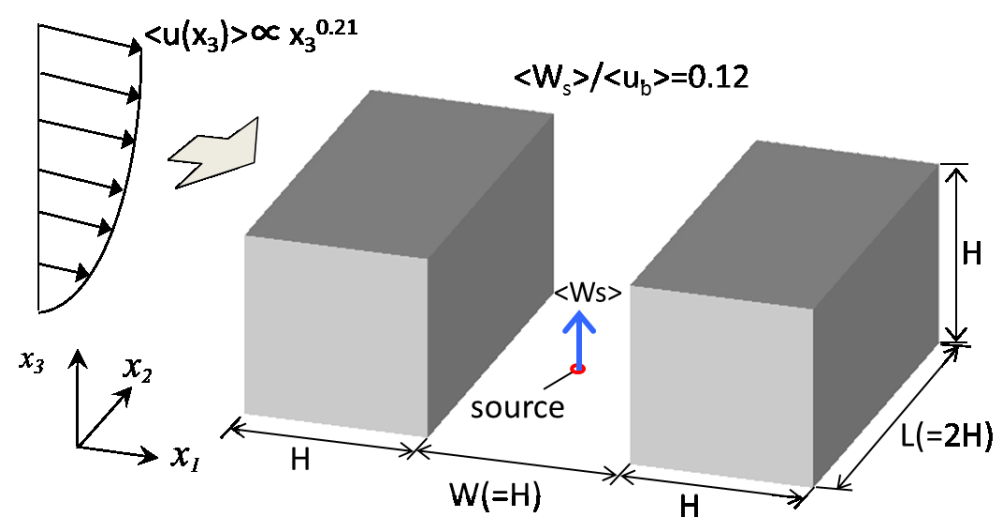

Fig. 1. Model configuration for this study.

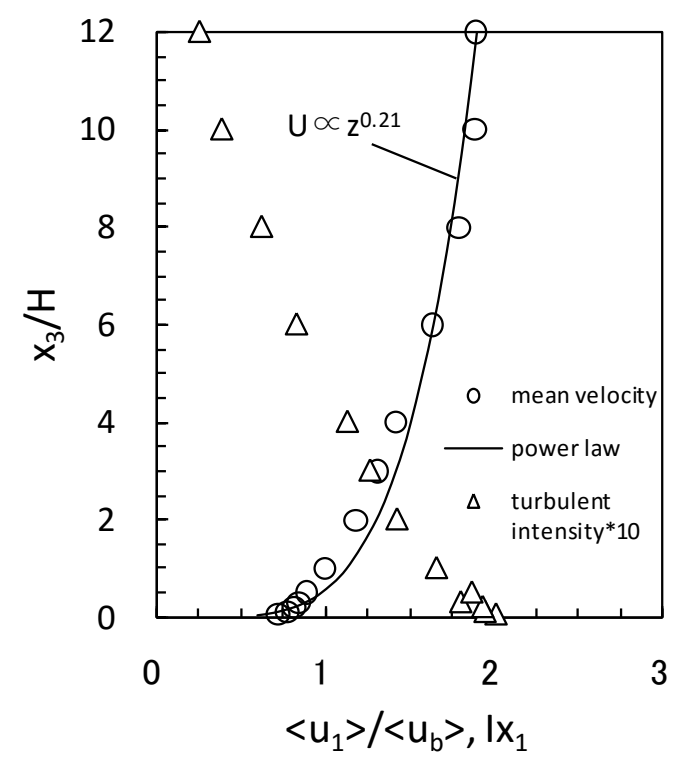

Fig. 2. Vertical profiles of mean velocity and turbulent intensity at inflow boundary. 


\section{$2.2 \quad$ Numerical methods}

The self-developed code used for both RANS and LES computations is based on a finite volume approach for solving flow and concentration equations on structured rectangular grids.

(1) RANS: The RNG model, which shows best agreement with the experiment of the four types of turbulence models in the previous study (Tominaga and Stathopoulos, 2009), was used. The turbulent Schmidt number was set to 0.7 (Tominaga and Stathopoulos, 2007). The QUICK scheme was used for discretizing momentum and concentration equations. Unsteady calculations were carried out, but results obtained by RNG showed almost no unsteady motion like vortex shedding.

(2) LES: The standard Smagorinsky model with the empirical constant $\mathrm{C}_{\mathrm{S}}=0.12$ was used for the sub-grid scale eddy viscosity model (Smagorisky, 1963). Applicability of the Smagorinsly model with $\mathrm{C}_{\mathrm{S}}=0.12$ for a flow around a building model was confirmed in the previous studies (Murakami, 1993; Tominaga et al., 2008a). Near the wall, the length scale was modified by a Van Driest (1956) damping function. The subgrid scale Schmidt number was set to 0.5 (Antonopoulos-Domis, 1981). It should be noted that it does not necessary that optimum values of Schmidt number are equal in LES and RANS, because their modeling length scales are completely different. We used the same values with those in the previous study (Tominaga and Stathopoulos, 2010) by considering their consistency. A second-order centered difference scheme was adopted for the spatial derivatives. For time advancement, the Adams-Bashforth scheme was used for the convection terms and the Crank-Nicolson scheme for the diffusion terms. Although the methods adopted in the LES computation were rather conventional, simple and traditional sub-grid scale models were used in order to focus only on the difference between the modeling approaches of RANS and LES, that is, whether transient flow can be solved or not. The time step is set to $\Delta \mathrm{t}=1.0-4 \mathrm{~s}$. Before time averaging, the computation was run during for 60 non-dimensional time units $\mathrm{t}^{*}(=\mathrm{t} \times<\mathrm{ub}>/ \mathrm{H})$ to remove the influence of an initial condition. Then, the computations were conducted for 200 non-dimensional time units $t^{*}$ $\left(=\mathrm{t} \times<\mathrm{u}_{\mathrm{b}}>/ \mathrm{H}\right)$, which is correspond to $5 \mathrm{~s}$ in real time scale, to determine the time-averaged values. It was confirmed that the statistical results are almost repeatable with a longer averaging period.

\subsection{Boundary conditions}

The boundary conditions were set by basically following the guidelines provided by AIJ (Tominaga et al., $2008 \mathrm{~b})$. The computational domain covered a volume of $18 \mathrm{H}\left(\mathrm{x}_{1}\right) \times 12 \mathrm{H}\left(\mathrm{x}_{2}\right) \times 6 \mathrm{H}\left(\mathrm{x}_{3}\right)$. This domain was discretized into $108\left(\mathrm{x}_{1}\right) \times 76\left(\mathrm{x}_{2}\right) \times 42\left(\mathrm{x}_{3}\right)$ grids. The minimum grid width was $0.0025 \mathrm{H}$. These conditions were the same in both computations. In the RNG computations, it was confirmed that the prediction results did not change significantly with the finer grids. Although the girds seem to be rather coarse for LES, the same grids with the RANS computation are intentionally used for the same reason why the conventional and simple LES modeling are adopted as mentioned before. Turbulence in the exhaust outlet velocity was not considered.

(1) RNG: The vertical distributions of $<\mathrm{u}_{1}>$, $\mathrm{k}$ and $\varepsilon$ at the inflow boundaries were based on the experiment. The generalized log law was used for the solid boundary (Launder and Spalding, 1974).

(2) LES: A separate LES computation of turbulent boundary layer flow was conducted to generate inflow turbulence. The inflow generating method used here was that proposed by Kataoka and Mizuno (2002). It was confirmed that this method implemented to our computational code works well for reproducing the velocity and turbulence intensity profiles in arbitrary wind tunnel experiments (Tomianga et al., 2008a; Tominaga and Stathopoulos, 2010). For the boundary condition at the solid walls, a linear or 1/7 power law distribution of instantaneous velocity was assumed (Werner and Wengle, 1991). In this method, a no-slip condition is employed when a first grid point within the viscous sub-layer, or else a 1/7 power-law profile is assumed.

\section{$3 \quad$ Results and Discussion}

\subsection{Flow fields}


Fig. 3 compares the velocity vectors on the vertical and horizontal planes. On the vertical section, recirculation flows were observed in the street canyons in the experiment and CFD. The experimental flow pattern in the street canyon is quite similar with the result of flow visualization for the same aspect ratio with this study $(\mathrm{W} / \mathrm{H}=1)$ in continued street canyons presented in Chang and Meroney (2003). Recirculation eddies in both RNG and LES formed in a region farther from the ground than in the experiment. The vertical component of velocity in the street canyon, which was rather small in LES, was dominant in RNG. The general pattern of the recirculation flow in LES was closer to that in the experiment than in RNG. On the other hand, on a horizontal section at $\mathrm{x}_{3} / \mathrm{H}=0.1$, two vortices appeared within the street canyons in both experiment and CFD. Recirculation eddies in RNG formed in a region farther from the source point than in LES. The strong secondary flow in the upwind region just in front of the source point, which was not observed in LES or the experiment, was observed in RNG. The horizontal velocity near the upwind building in RNG was much stronger than that in LES. As discussed later, this stronger horizontal velocity contributes to the transport of pollutant outward from the center region in the canyon.

The distributions of turbulent energy $\mathrm{k}$ in the street canyon on vertical and horizontal planes are illustrated in Fig. 4. A peak of k, which is over 5 times larger than the spatial averaged value, is observed in front of the downwind building in LES, but there is no peak in RNG. The value of $\mathrm{k}$ given by LES is larger than that given by RNG over the whole area of the street canyon. These results indicate that turbulent momentum diffusion in the street canyon was supplied from the upper region into the street canyon. This smaller value of $\mathrm{k}$ in the street canyon given by RNG made the recirculation flow larger than that given by LES, because the momentum diffusion becomes small in RNG.

The tendency of RNG to show larger velocity and smaller k values than LES is also observed in the results obtained by the previous study for the cubic building case (Tominaga and Stathopoulos, 2010). However, for the street canyon, the difference between models becomes large, since the horizontal component of velocity in the street canyon is larger than that on the roof of the cubic building.

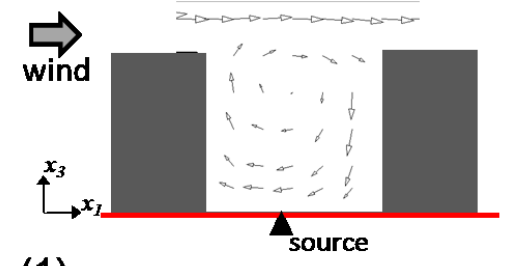

(1)

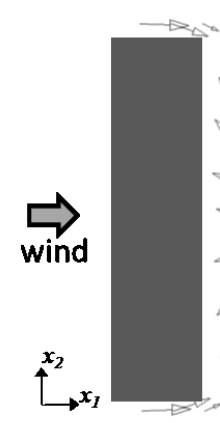

(2)
EXP.

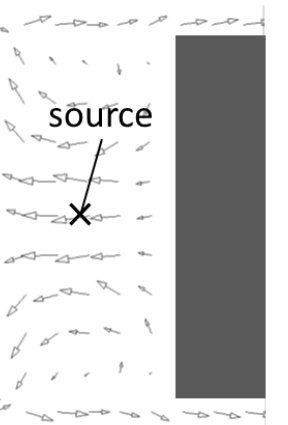

EXP.

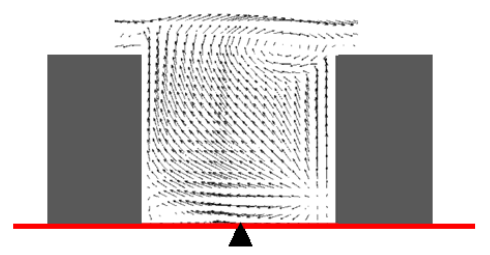

RNG

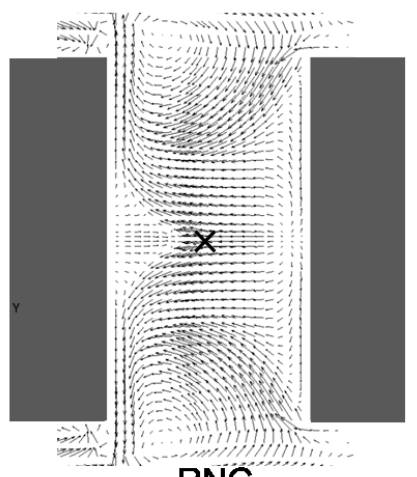

RNG

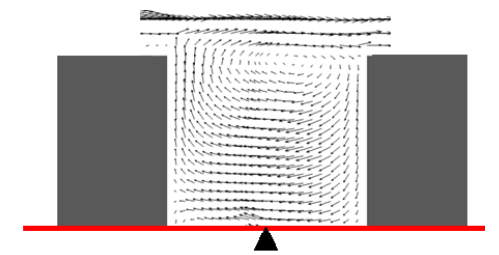

LES

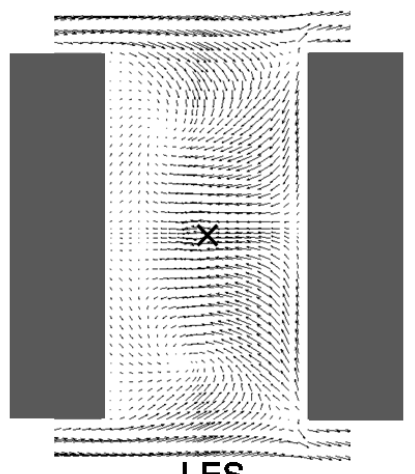

LES

Fig. 3. Comparisons of velocity vectors on vertical and horizontal planes in a street canyon; (1) Vertical plane (central section), (2) Horizontal plane ( $\left.\mathrm{x}_{3} / \mathrm{H}=0.1\right)$. 

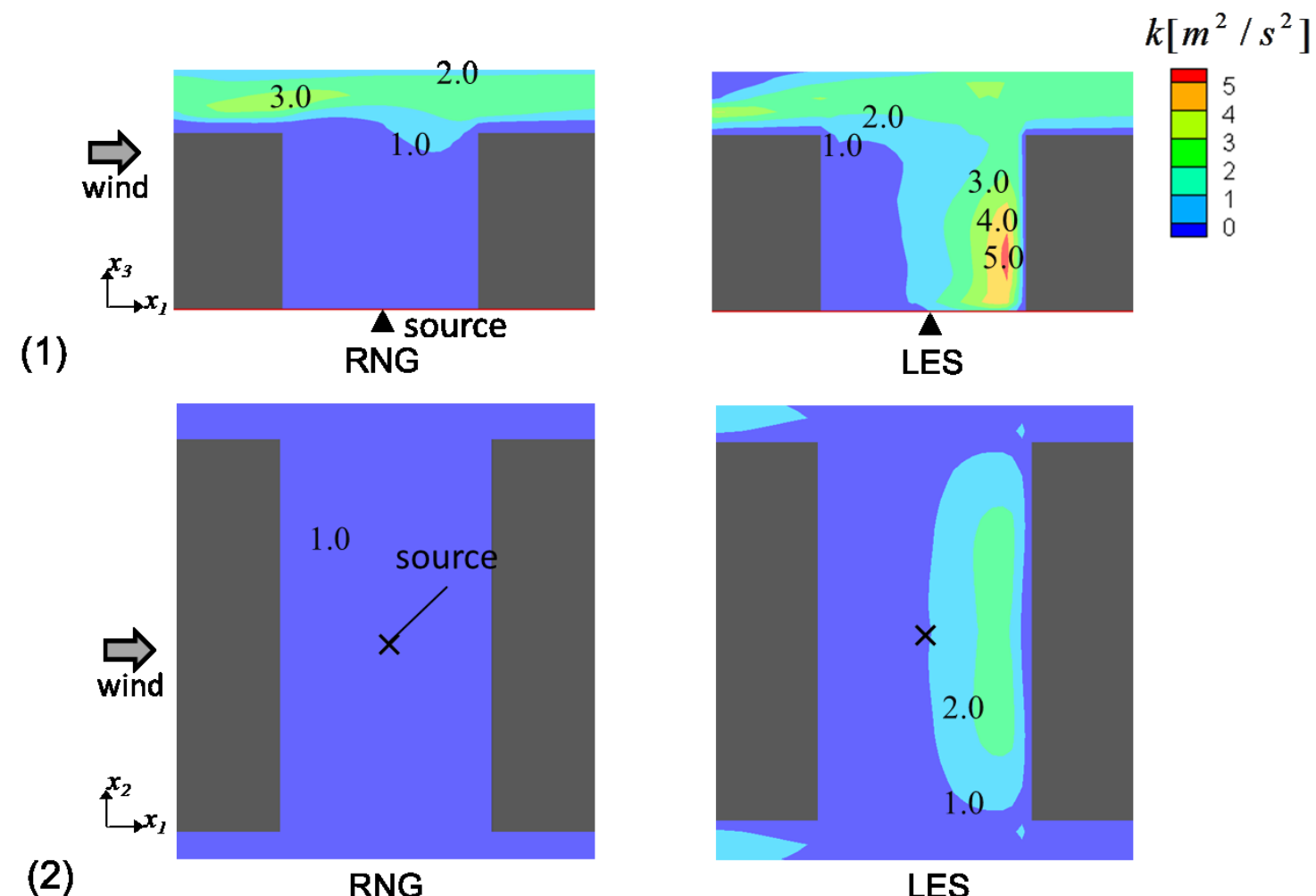

Fig. 4. Comparisons of turbulent energy k on vertical and horizontal planes in a street canyon; (1) Vertical plane (central section), (2) Horizontal plane $\left(\mathrm{x}_{3} / \mathrm{H}=0.1\right)$.

\subsection{Time-averaged concentration}

Fig. 5 compares the contours of the time-averaged concentration $<c>$ on vertical and horizontal planes obtained from CFD and the experiment. On the vertical plane, a high concentration region spread to the leeward side of the upwind building in both models. These high concentrations were mainly transferred by recirculation flows in this region (cf. Fig. 2). This tendency can be also observed in the experimental results with the $\mathrm{W} / \mathrm{H}=1$ case reported by Chang and Meroney (2003). The transfer concentration in the upwind direction by advection was dominant in the result by RNG. However, the diffusion of concentration in the vertical direction above the source point in RNG was smaller than that in LES and the experiment. On the horizontal plane, the distribution patterns for the two models were very different. RNG showed larger concentrations in the region upwind of the source point than LES. The high concentration region of $<\mathrm{c}>$ in RNG also expanded horizontally near the leeward side of the upwind building in comparison with LES and the experiment. Generally, RNG underestimated the turbulence diffusion in the street canyon. On the other hand, in LES, the concentrations were widely spread in the horizontal direction. The general distribution of $\langle\mathrm{c}>$ from the experiment is well reproduced in LES than RNG.

The distribution of $<\mathrm{c}>$ along-the streamwise direction $\left(\mathrm{x}_{1}\right)$ passing through the source in the street canyon is shown in Fig. 6. Error bars indicate \pm standard deviation of replicate measurements within an experiment. At the bottom height of the canyon, in the upwind area from the source point, the RNG values were approximately 4 times higher than the LES values in maximum. The distribution of $<\mathrm{c}>$ obtained by the experiment is well reproduced by LES. At the middle and the top height of the canyon, RNG shows 3 times higher value of $<c>$ near the leeward wall of the upwind building than LES and the experiment, which gives rather flat distribution. In general, the distributions of $<c>$ obtained by LES show good agreement with the experiment (approximately $25 \%$ on average). These results were caused by larger turbulence diffusions in lateral direction obtained by LES in comparison with RNG (cf. Fig. 5). On the time-averaged concentration, the effect of horizontal advection on concentration in LES is larger than that in RNG. This tendency is similar in the cubic 
building (Tominaga and Stathopoulos, 2010) and the street canyon cases. However, the difference between two approaches appeared in the street canyon is larger than that in the flow around the cube. This is because the scalar transport in the horizontal direction inside the street canyon is more dominant than that on the roof of the cubic building.

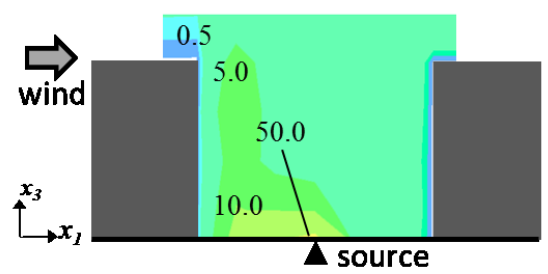

(1)

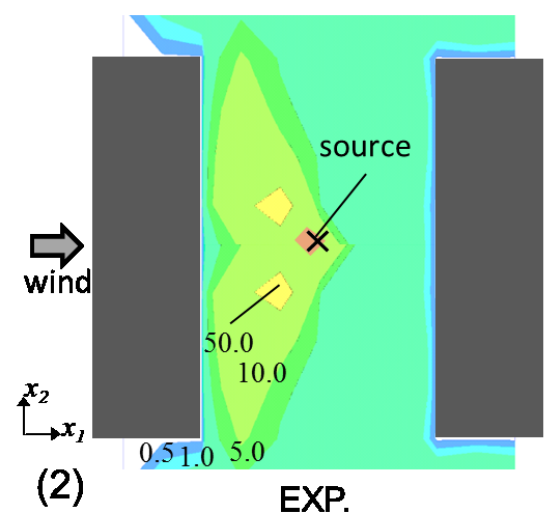

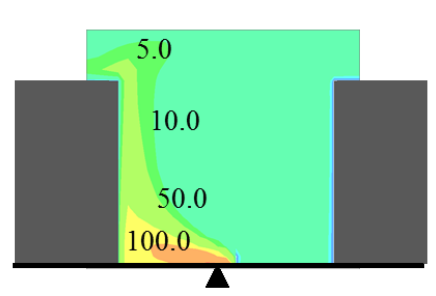

RNG

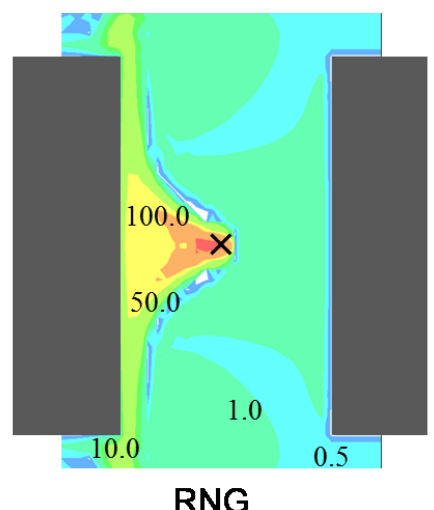

$<c>[p p m]$

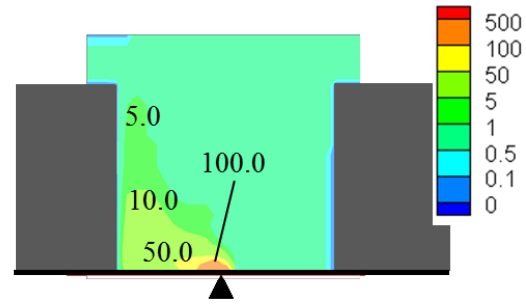

LES

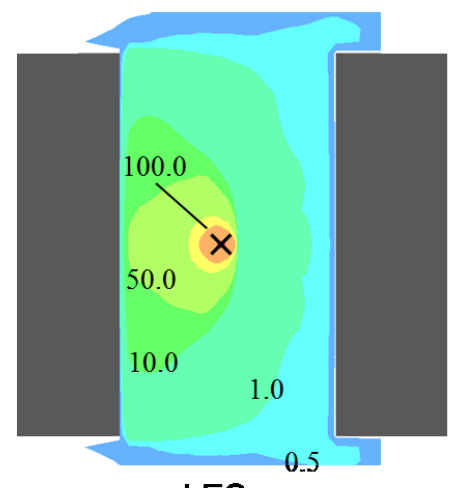

LES

Fig. 5. Comparisons of time-averaged concentration $<\mathrm{c}>$ on vertical and horizontal planes in a street canyon; (1) Vertical plane (central section), (2) Horizontal plane ( $\left.\mathrm{x}_{3} / \mathrm{H}=0.1\right)$. 

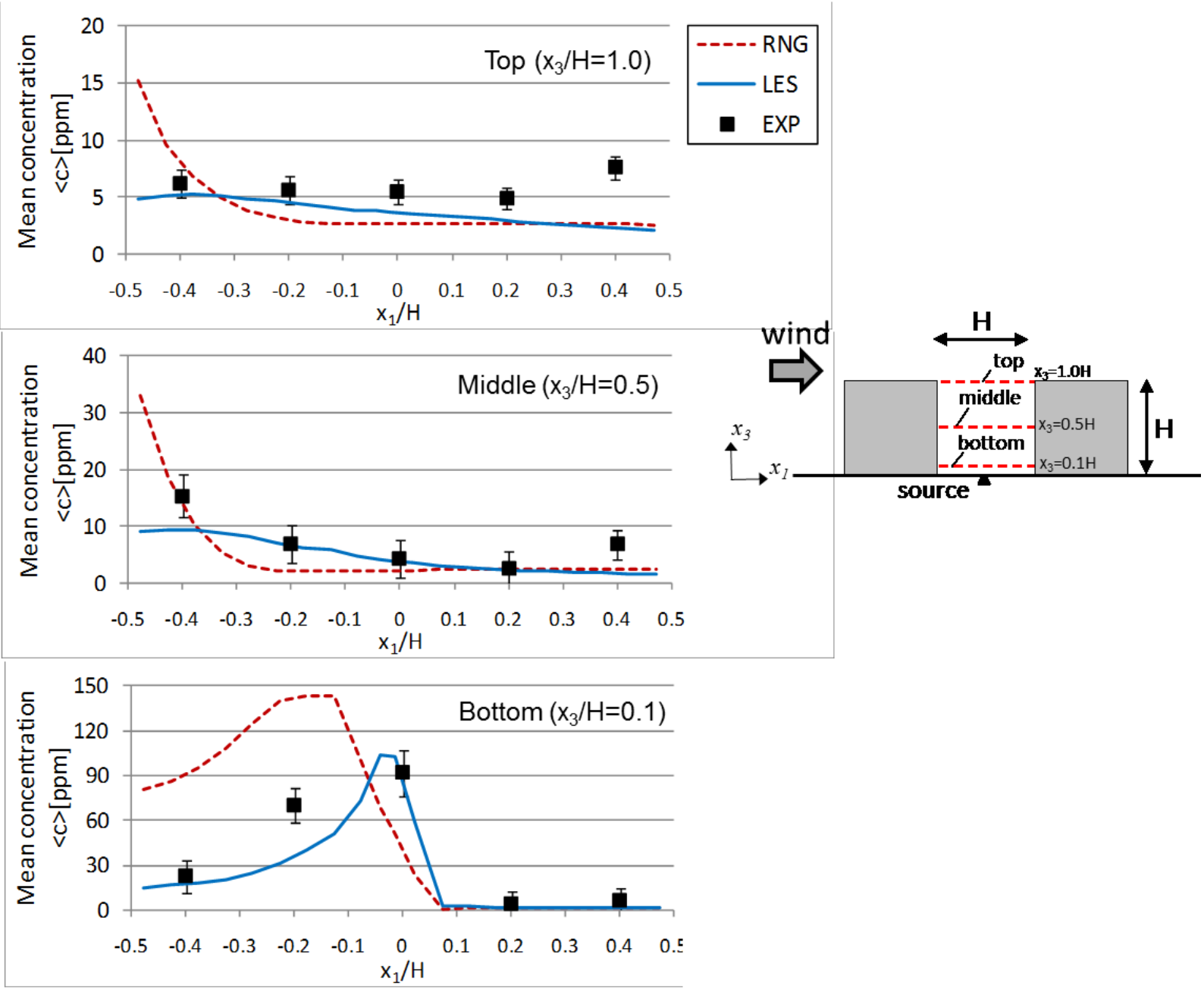

Fig. 6. Distributions of time-averaged concentration $<\mathrm{c}>$ inside a street canyon at three different heights (center line).

\subsection{Concentration fluctuations}

Fig. 7 shows the time history of instantaneous concentration inside the street canyon with three different heights at the centerline obtained by LES. The values are normalized by the time averaged concentration at the same points. Results are presented for different dimensionless time steps $t *$ defined by $\left\langle\mathrm{u}_{b}>\right.$ and $\mathrm{H}$, as mentioned previously. Even at the top of the canyon, high concentrations, which are approximately 5 times larger than the time-averaged value in maximum, occur intermittently. The time series iso-surfaces of instantaneous concentration in street canyon are illustrated in Fig. 8. The shapes of the high concentration region vary widely in each time step. These time series assert that the plume dispersion in the street canyon was indeed highly unsteady. The r.m.s. value of concentration fluctuation predicted by LES is compared with the experimental data at the bottom of the street canyon in Fig. 9. The values predicted by LES are in good agreement with the experimental data. The r.m.s. value shows a large peak above the source point, and the value is relatively low in downward region from the source. The tendency that the distribution of r.m.s. values tends to be similar to that of the mean concentration was also observed for the cubic building case. 


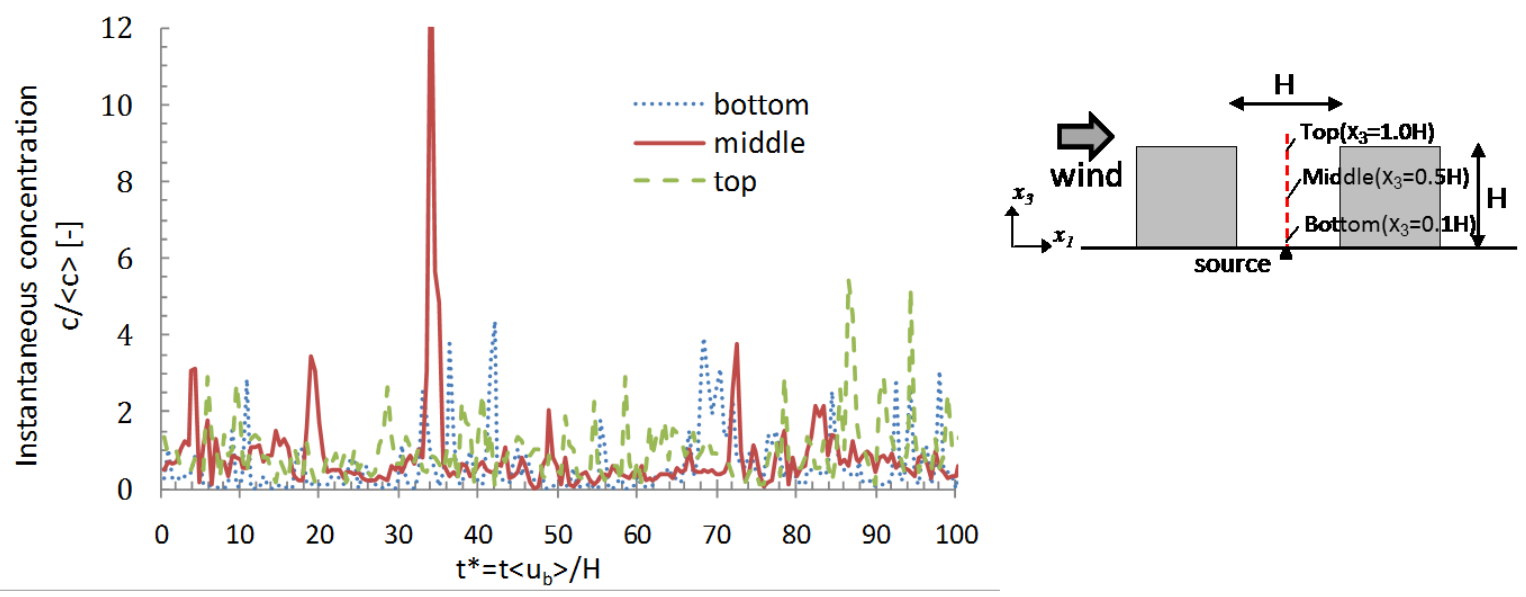

Fig. 7. Time history of instantaneous concentration c inside a street canyon at three different heights (center line) obtained by LES.

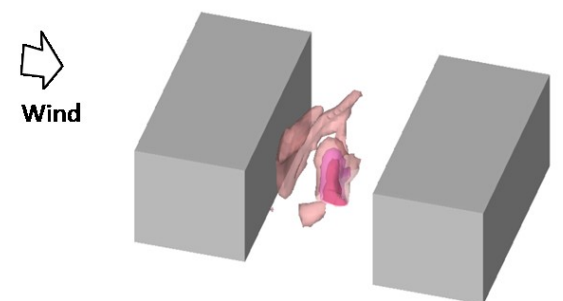

$t^{*}=0.0$
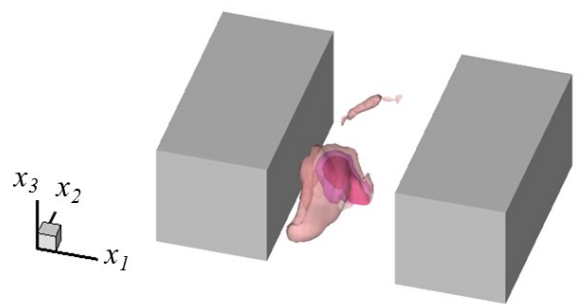

$t^{*}=30.0$

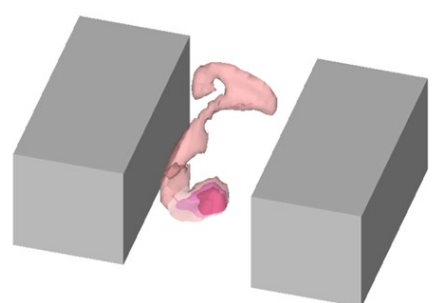

$t^{\star}=15.0$

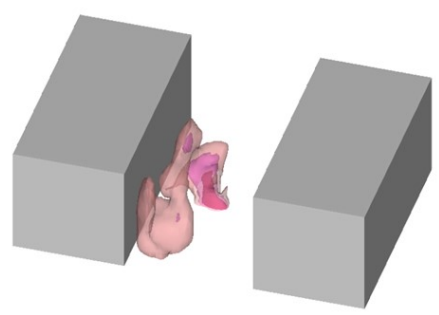

$t^{*}=45.0$

Fig. 8. Time series of iso-surfaces of instantaneous concentration c inside a street canyon obtained by LES (Deep, medium and light colors of iso-surfaces indicate 200ppm, 100ppm and 20ppm, respectively). 


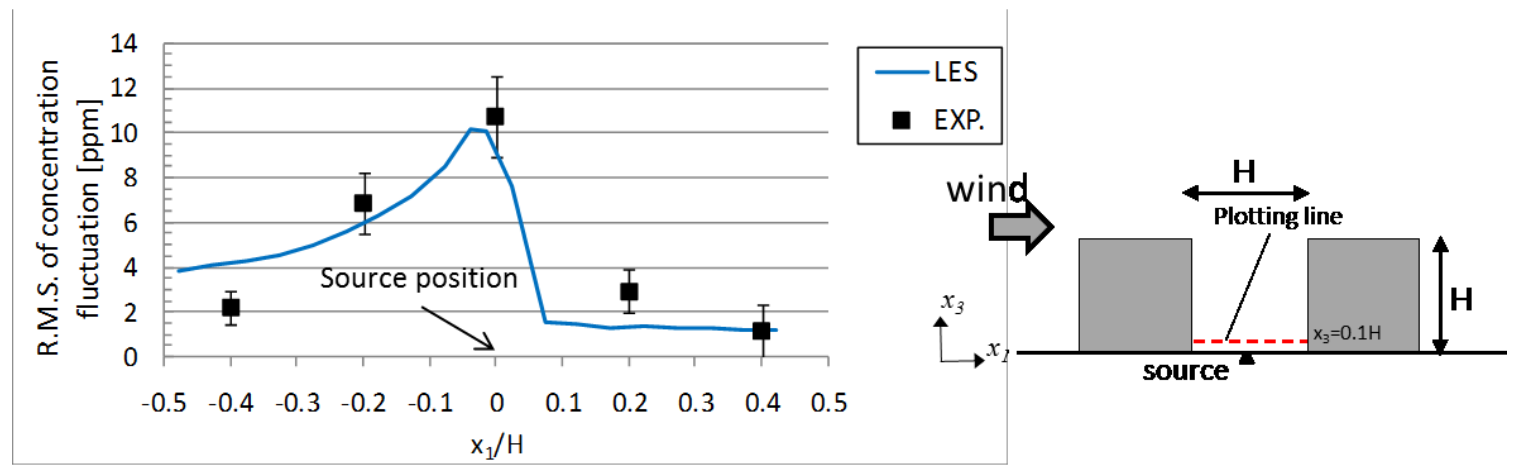

Fig. 9. Distribution of r.m.s. value of concentration $\sqrt{\left\langle c^{\prime 2}\right\rangle}$ inside a street canyon across a source point (center line).

\subsection{Concentration fluxes}

The distribution of concentration fluxes represents the essentials of concentration transport, because it can provide very important information for investigating the validity of a model used for concentration transport. Scalar transport of concentration consists of convective and turbulent diffusion effects, which are expressed by convection as mean scalar fluxes $\left.\left\langle\mathrm{u}_{\mathrm{i}}\right\rangle<\mathrm{c}\right\rangle$ and turbulent diffusion fluxes $\left\langle\mathrm{u}_{\mathrm{i}}{ }^{\prime} \mathrm{c}{ }^{\prime}\right\rangle$, respectively. The convective fluxes can be estimated by using mean velocities $<\mathrm{u}_{\mathrm{i}}>$ and mean concentration $<\mathrm{c}>$. Although the turbulent diffusion fluxes are calculated directly in LES, they are modeled by the gradient diffusion hypothesis,

$-<u_{i}{ }^{\prime} c^{\prime}>=\frac{v_{t}}{S c_{t}} \frac{\partial<c>}{\partial x_{i}}$

where $v_{t}$ is the eddy viscosity and $\mathrm{Sc}_{\mathrm{t}}$ is the turbulent Schmidt number, in RNG. Although the turbulent scalar fluxes determined by this model can be changed with the Sct as a matter of course, the values actually used in the computation are intentionally compared in this study.

Fig. 10 (1) compares the vertical components of turbulent diffusion flux $<\mathrm{u}_{3}$ ' $\mathrm{c}^{\prime}>$ on the vertical section. The values in the region upwind of the source point are larger in RNG than those in LES. On the other hand, the LES results show large positive values in the upper region above the source point, which are rather small in RNG. The lateral components of turbulent diffusion flux $\left\langle\mathrm{u}_{2}\right.$ ' $\left.{ }^{\prime}\right\rangle$ on the horizontal plane are shown in Fig. 10 (2). A significant difference between the two models is observed. The LES results show large values of flux more widely spread in the lateral directions than those of RNG. Consequently, LES shows a much larger contribution of turbulent diffusion fluxes than RNG. The large difference between the modeled turbulent fluxes suggests that the accuracy of the turbulent diffusion modeling is very important in predicting the mean concentration distribution. 

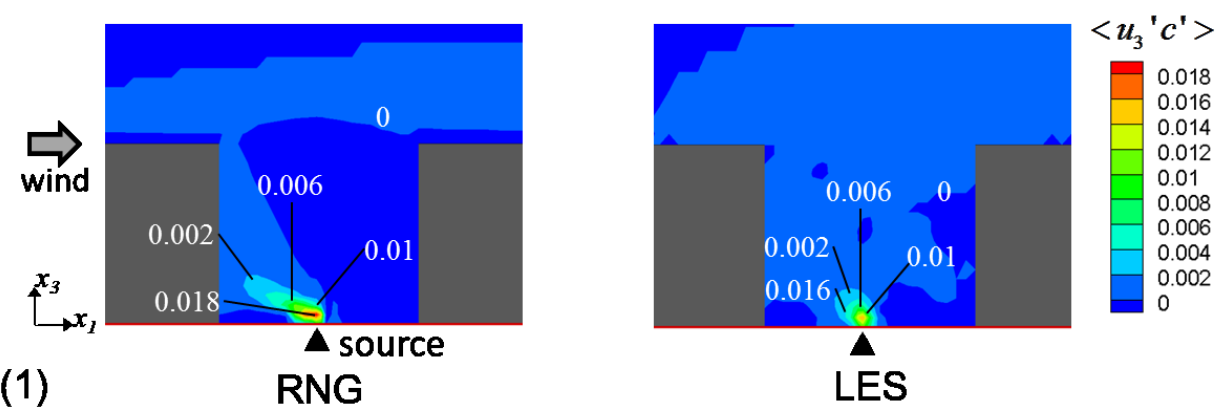

(1)

RNG

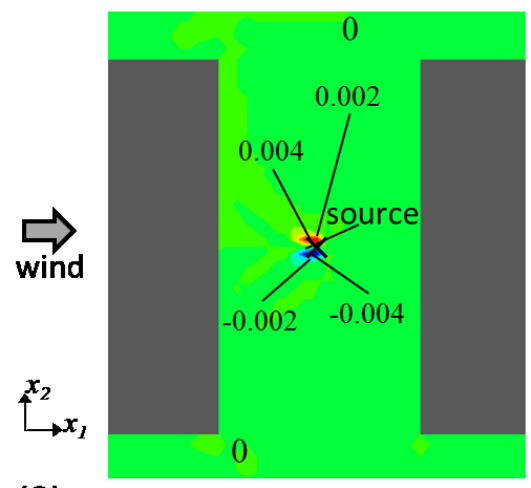

(2)

RNG

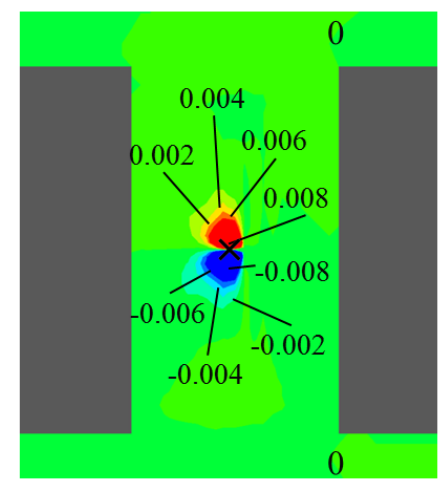

$<u_{2}{ }^{\prime} c^{\prime}>$

0.008

0.006
0.004
0.002

0.002

\begin{tabular}{l}
0 \\
\hline-0.002
\end{tabular}

$-0.004$

-0.006
-0.008

Fig. 10. Distributions of turbulent diffusion flux in a street canyon; (1) $<\mathrm{u}_{3}$ 'c'> on vertical plane at center section, (2) $<\mathrm{u}_{2}$ 'c'> on horizontal plane $\left(\mathrm{x}_{3} / \mathrm{H}=0.1\right)$.

\section{$4 \quad$ Conclusions}

CFD modeling using RANS and LES of pollutant dispersion in a three-dimensional street canyon is investigated by comparison with measurements. Clearly, LES computation can provide important information on instantaneous fluctuations of concentration, which cannot be obtained by RANS computations (RNG). The results obtained by LES indicate that the plume dispersion in the street canyon is highly unsteady. Moreover, in terms of the difference of turbulent scalar diffusion modeling by two modeling approaches, the following conclusions can be made from this study:

(1) Simple LES modeling gives better results than conventional RANS computation (RNG) modeling of the distribution of mean concentration in comparison with the wind tunnel experiment. For example, at the bottom height of the canyon, in the upwind area from the source point, the RNG values were approximately 4 times higher than the LES and the experimental values in maximum. The horizontal diffusion of concentration is well reproduced by LES, due mainly to the reproduction of unsteady concentration fluctuations in the street canyon.

(2) On the other hand, RNG underestimates turbulence diffusion in the street canyon in comparison with LES. The difference of the scalar transport properties between two approaches can be explained clearly by the difference of turbulence scalar flux modeling. The turbulence diffusion flux obtained by LES is more widely spread in the lateral directions than those by RNG. The modeling accuracy of turbulent diffusion is very important for predicting the concentration distribution.

(3) Wind flow and concentration fields around a street canyon and a cubic building show similar variation trends when computed by using LES or RANS methodologies. However, the difference between two approaches appeared in the street canyon is larger than that in the flow around the cube. This is because the scalar transport in the horizontal direction inside the street canyon is more dominant than that on the roof of the cubic building. 
To generalize the above conclusions, further investigations are required on the influence of canyon shape on the flow regime around the street canyon and the effect of the surroundings, which is closely related to the turbulence intensity of the flow upstream and inside the street canyon.

\section{Nomenclature}

c : concentration

$<f$ : ensemble average

$\mathrm{H} \quad$ : building height

$\mathrm{k} \quad$ : turbulent energy

$\mathrm{L} \quad$ : building length

$t^{*} \quad$ : non-dimensional time unit $\left(=\mathrm{t}<\mathrm{u}_{\mathrm{b}}>/ \mathrm{H}\right)$

$\mathrm{u}_{\mathrm{i}} \quad$ : three components of wind velocity

$\mathrm{u}_{\mathrm{b}} \quad$ : inflow velocity at building height $\mathrm{H}$

$\mathrm{W}$ : distance between buildings

$\mathrm{w}_{\mathrm{s}} \quad$ : exit velocity

$x_{i} \quad$ : three components of spatial coordinates $(i=1,2,3$ : streamwise, lateral, vertical $)$

\section{Acknowledgements}

This paper is an extension of the earlier study conducted by the authors (Tominaga and Stathopoulos, 2009; Tominaga and Stathopoulos, 2010), which started during the period the first author worked as a visiting researcher in the Department of Building, Civil and Environmental Engineering of Concordia University, Montreal, Canada (September 2006 to February 2007). The authors would like to express their gratitude to Niigata Institute of Technology for supporting this stay. The first author also gratefully acknowledges the support provided for this research by Grants-in-Aid for Scientific Research in Japan (No. 21560622) and the Obayashi Foundation.

\section{References}

Antonopoulos-Domis, M., 1981. Large eddy simulation of a passive scalar in isotropic turbulence, Journal of Fluid Mechanics 104, 55-79.

Baik, J.-J., Kim, J.-J. and Fernando, H.J.S., 2003. A CFD model for simulating urban flow and dispersion. Journal of Applied Meteorology 42, 1636-1648.

Blocken, B., Stathopoulos, T., Saathoff, P., Wang X., 2008. Numerical evaluation of pollutant dispersion in the built environment: Comparisons between models and experiments. Journal of Wind Engineering and Industrial Aerodynamics 96, 1817-1831.

Britter, R., Schatzmann, M., Eds., 2007. Model evaluation guidance and protocol document. COST, Brussels.

Chan, A.T., So, E.S.P., Samad, S.C., 2001. Strategic guidelines for street canyon geometry to achieve sustainable street air quality. Atmospheric Environment 35 (32), 5681-5691.

Chan, T.L., Dong, G., Leung, C.W., Cheung, C.S., Hung, W.T., 2002. Validation of a two-dimensional pollutant dispersion model in an isolated street canyon. Atmospheric Environment 36, 861-872.

Chang, C-H., Meroney, R. N., 2001, Numerical and physical modeling of bluff body flow and dispersion in urban street canyons. Journal of Wind Engineering and Industrial Aerodynamics 89, 1325-1334.

Chang, C-H., Meroney, R. N., 2003, Concentration and flow distributions in urban street canyons: wind tunnel and computational data. Journal of Wind Engineering and Industrial Aerodynamics 91, 1141-1154.

Hanna, S. R., Hansen, O. R., Dharmavaram, S., 2004, FLACS CFD air quality model performance evaluation with Kit Fox, MUST, Prairie Grass, and EMU observations, Atmospheric Environment 38, 4675-4687.

Harms, F., Leitl, B., Schatzmann, M., 2005. Comparison of tracer dispersion through a model of an idealized urban area from field (MUST) and wind tunnel measurements. Proceedings International Workshop on Physical Modelling of Flow and Dispersion Phenomena, London, Canada.

Hu L.H., Huo R., Yang D., 2009. Large eddy simulation of fire-induced buoyancy driven plume dispersion in an urban street canyon under perpendicular wind flow. Journal of Hazardous Materials 166, 394-406.

Hunter, L. J., Johnson, G. T. and Watson, L. D., 1992. An investigation of three-dimensional characteristics of flow regimes within the urban canyon. Atmospheric Environment 26B(4), 425-432. 
Kataoka, H., Mizuno, M., 2002. Numerical flow computation around aeroelastic 3D cylinder using inflow turbulence, Wind and Structures 5, 379-292.

Kim, J.-J., Baik, J.-J., 2003. Effects of inflow turbulence intensity on flow and pollutant dispersion in an urban street canyon. Journal of Wind Engineering and Industrial Aerodynamics 91, 309-329.

Launder, B. E. and Spalding, D. B., 1972. Mathematical models of turbulence. Academic Press, New York.

Launder, B.E., Spalding, D.B., 1974. The numerical computation of turbulent flows. Comput. Methods Appl. Mech. Eng. 3, 269-289.

Li, X.-X., Liu, C.-H., Leung, D. Y. C., Lam, K. M., 2006. Recent progress in CFD modelling of wind field and pollutant transport in street canyons. Atmospheric Environment 40, 5640-5658.

Li X.-X., Liu C.-H., Leung D.Y.C., 2008. Large-eddy simulation of flow and pollutant dis-persion in highaspect-ratio urban street canyons with wall model. Boundary-Layer Meteorology 129, 249-268.

Meroney, R. N., Pavageau, M., Rafailidis, S., Schatzmann, M., 1996, Study of line source characteristics for 2-D physical modelling of pollutant dispersion in street canyons. Journal of Wind Engineering and Industrial Aerodynamics 62, 37-56.

Milliez, M., Carissimo, B., 2007. Numerical simulations of pollutant dispersion in an idealized urban area, for different meteorological conditions. Boundary-Layer Meteorology 122, 321-342.

Murakami, S., 1993. Comparison of various turbulence models applied to a bluff body. Journal of Wind Engineering and Industrial Aerodynamics 46-47, 21-36.

Nazridoust, K., Ahmadi, G., 2006, Airflow and pollutant transport in street canyons. Journal of Wind Engineering and Industrial Aerodynamics 94, 491-522.

Pavageau, M., Schatzmann, M., 1999. Wind tunnel measurements of concentration fluctuations in an urban street canyon. Atmospheric Environment 33, 3961-3971.

Rodi, W., 1997, Comparison of LES and RANS calculations of the flow around bluff bodies. Journal of Wind Engineering and Industrial Aerodynamics 69-71, 55-75.

Santiago, J. L., Dejoan, A., Martilli, A., Martin, F, Pinelli, A., 2010, Comparison Between Large-Eddy Simulation and Reynolds-Averaged Navier-Stokes Computations for the MUST Field Experiment. Part I: Study of the Flow for an Incident Wind Directed Perpendicularly to the Front Array of Containers, Boundary-Layer Meteorology 135, 109-132.

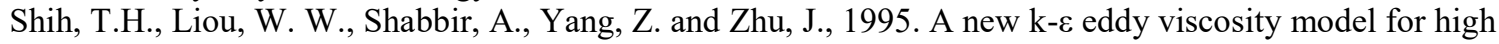
Reynolds number turbulent flows. Computers and Fluids 24, 227-238.

Sini, J.-F., Anquetin, S., Mestayer, P.G., 1996. Pollutant dispersion and thermal effects in urban street canyons. Atmospheric Environment 30, 2659-2677.

Smagorinsky, J., 1963. General circulation experiments with the primitive equations I. The basic experiment. Monthly Weather Review 91, 99-164.

Tominaga, Y. and Stathopoulos, T., 2007. Turbulent Schmidt numbers for CFD analysis with various types of flowfield. Atmospheric Environment 41, 8091-8099.

Tominaga, Y., Mochida, A., Murakami, S., Sawaki, S., 2008a. Comparison of various revised k- $\varepsilon$ models and LES applied to flow around a high-rise building model with 1:1:2 shape placed within the surface boundary layer. Journal of Wind Engineering and Industrial Aerodynamics 96, 389-411.

Tominaga, Y., Mochida, A., Yoshie, R., Kataoka, H., Nozu, T., Yoshikawa, M., Shirasawa, T., 2008b, AIJ guidelines for practical applications of CFD to pedestrian wind environment around buildings, Journal of Wind Engineering and Industrial Aerodynamics 96, 1749-1761.

Tominaga, Y., Stathopoulos, T., 2009. Numerical simulation of dispersion around an isolated cubic building : Comparison of various types of k- $\varepsilon$ models. Atmospheric Environment 43, 3200-3210.

Tominaga, Y., Stathopoulos, T., 2010, Numerical simulation of dispersion around an isolated cubic building: Model evaluation of RANS and LES, Building and Environment 45, 2231-2239.

Van Driest, E. R., 1956, On turbulent flow near a wall, Journal of Aeronautical Science 23: 11, 1007-1011.

Vardoulakis, S., Fisher, B.E., Pericleous, K., Gonzalez-Flesca, N., 2003. Modelling air quality in street canyons: a review. Atmospheric Environment 37, 155-182.

Walton, A., Cheng, A.Y.S., Yeung, W.C., 2002. Large-eddy simulation of pollution dispersion in an urban street canyon - Part I: comparison with field data. Atmospheric Environment 36, 3601-3613.

Walton, A., Cheng, A.Y.S., 2002. Large-eddy simulation of pollution dispersion in an urban street canyon Part II : idealised canyon simulation. Atmospheric Environment 36, 3615-3627.

Werner, H., Wengle, H., 1991. Large eddy simulation of turbulent flow over and around a cube in plane channel. In : Proceedings of 8th Symposium on Turbulent Shear Flows, 155-168. 
Xie, X. , Huang, Z., Wang, J., Xie, Z., 2005, The impact of solar radiation and street layout on pollutant dispersion in street canyon. Building and Environment 40, 201-212.

Yakhot, V., Orszag, S.A., Thangam, S., Gatski, T.B., Speziale, C.G., 1992. Development of turbulence models for shear flows by a double expansion technique. Physics of Fluids A4, 1510-1520.

Yee E, Biltoft C. A., 2004. Concentration fluctuation measurements in a plume dispersing through a regular array of obstacles. Boundary-Layer Meteorology 111, 363-415. 